\title{
EFECTO DE LA PROTECCIÓN Y LA NUTRICIÓN SOBRE PARÁMETROS HÍDRICOS Y EL RENDIMIENTO DE CULANTRO COYOTE (Eryngium foetidum) HIDROPÓNICO
}

\author{
Freddy Soto-Bravo ${ }^{1 / *}$, Gustavo Alonso Rodríguez-Ocampo ${ }^{2}$ \\ Palabras clave: Manejo del riego; evapotranspiración; volumen de drenaje; eficiencia de uso de agua; \\ rendimiento de cultivo. \\ Keywords: Irrigation management; evapotranspiration; drainage volume; water use efficiency; crop productivity.
}

Recibido: 15/07/2019

Aceptado: 12/11/2019

RESUMEN

Introducción. En Costa Rica, las prácticas de cultivo en culantro coyote convencional, en suelo a campo abierto, hacen ineficiente el uso de los recursos y reducen la calidad, inocuidad y los rendimientos, por lo que una alternativa es el cultivo hidropónico protegido. Objetivo. El objetivo de este estudio fue evaluar el efecto de la protección y la nutrición sobre parámetros hídricos: eficiencia de uso de agua (EUA), evapotranspiración de cultivo $\left(\mathrm{ET}_{\mathrm{c}}\right)$, drenaje, coeficientes de cultivo $\left(\mathrm{K}_{\mathrm{c}}\right)$ y el rendimiento fresco de culantro coyote hidropónico. Materiales $\mathbf{y}$ métodos. El estudio se realizó en el 2016, en la Estación Experimental Agrícola Fabio Baudrit Moreno, Alajuela, Costa Rica. Comprendió 2 etapas de un cultivo, etapa I, en época seca protegido con sarán y etapa II, en época lluviosa protegido con plástico. En ambas etapas hubo 4 tratamientos que combinaron 2 condiciones ambientales, una con protección (CP) y otra sin protección (SP), con 2 niveles de nutrición:

\footnotetext{
* Autor para correspondencia. Correo electrónico: freddy.sotobravo@ucr.ac.cr

1 Universidad de Costa Rica, Estación Experimental Agrícola Fabio Baudrit Moreno, Costa Rica. (D) 0000-0003-1959-9597.
}

\section{ABSTRACT}

Effect of protection and the nutrition on water parameters and yield in hydroponic coriander wild (Eryngium foetidum). Introduction. In Costa Rica, cultivation practices in conventional wild coriander in open field soil, make resource use inefficient and reduce quality, safety and yields; therefore, an alternative is the protected hydroponic crop. Objective. The objective of this study was to evaluate the effect of protection and nutrition on water parameters: water use efficiency (WUE), crop evapotranspiration $\left(\mathrm{ET}_{\mathrm{c}}\right)$, drainage, crop coefficients $\left(\mathrm{K}_{c}\right)$ and the fresh yield of hydroponic wild coriander. Materials and methods. The study was conducted in 2016, at the Fabio Baudrit Moreno Agricultural Experimental Station, Alajuela, Costa Rica. It included 2 stages of a crop, stage I, in dry season protected with sarán and stage II in rainy season protected with plastic. In both stages there were 4 treatments that combined 2 environmental conditions,

2 Universidad de Costa Rica, Estación Experimental Agrícola Fabio Baudrit Moreno, Costa Rica. (D) 0000-0001-6033-684X. 
alta (NA) y baja concentración de nutrientes (NB). Se utilizó un diseño de 5 bloques al azar con arreglo bifactorial 2 × 2 . Resultados. El rendimiento fue similar en CP y SP, sin embargo, una menor $\mathrm{ET}_{\mathrm{c}}$ en $\mathrm{CP}$ incrementó la EUA. La $\mathrm{ET}_{\mathrm{c}}$ fue similar en ambos niveles de nutrición, no obstante, debido a mayor rendimiento, la EUA en NA fue superior. En etapa I, el drenaje únicamente fue afectado por la nutrición, por lo que fue menor con NB; en etapa II, el drenaje disminuyó apreciablemente con techo plástico (CP). Los $\mathrm{K}_{\mathrm{c}}$ no fueron afectados por ambos factores, obtuvieron valores máximos de 1,15 y 1,4 en etapas I y II, repectivamente. Conclusión. El sistema de cultivo hidropónico representa una excelente alternativa al sistema de producción convencional en suelo y campo abierto, ya que incrementó la EUA producto de una apreciable reducción de la $\mathrm{ET}_{\mathrm{c}}$ bajo protección y un sustancial incremento en los rendimientos con nivel de nutrición alto.

\section{INTRODUCCIÓN}

Eryngium foetidum L. (UmbelliferaeApiaceae), es conocido comúnmente como culantro mexicano, coyote, silvestre, espinoso, entre otros. Tradicionalmente ha sido utilizado como especia, fines medicinales y culinarios. Esta planta es originaria de zonas tropicales de América, entre Panamá, México, el archipiélago del Caribe y las Indias Occidentales (Santos 2001). En el siglo XV la población europea lo llevaron a otras zonas tropicales de todos los continentes (Morales et al. 2013). Actualmente, es un cultivo de gran importancia internacional, cultivado en Puerto Rico, República Dominicana, Cuba, islas de las Antillas, América Central, México, Brasil, Tailandia, Vietnam, Bangladesh y la India (Morales et al. 2013). En Costa Rica se cultiva desde 1990 para exportación, cuya demanda a Estados Unidos y Canadá ha incrementado en one with protection $(\mathrm{CP})$ and the other unprotected (SP), with 2 levels of nutrition: high (NA) and low nutrient concentration (NB). A randomized 5 blocks design with bifactorial arrangement of 2 x 2 was used. Results. The yield was similar in $\mathrm{CP}$ and SP, however, a lower $\mathrm{ET}_{\mathrm{c}}$ in $\mathrm{CP}$ increased the WUE. The $\mathrm{ET}_{\mathrm{c}}$ was similar in both levels of nutrition, however, due to higher yield, the WUE in NA was superior. In stage I, drainage was only affected by nutrition, being lower with NB; In stage II, drainage decreased significantly with plastic roof (CP). The $\mathrm{K}_{\mathrm{c}}$ were not affected by both factors, obtaining maximum values of 1.15 and 1.4 in stages I and II, respectively. Conclusion. The hydroponic cultivation system represents an excellent alternative to the conventional production system in soil and open field, since it increased the WUE, product of an appreciable reduction of the $\mathrm{ET}_{\mathrm{c}}$ under protection and a substantial increase in yields with high nutrition level.

los últimos años (Eunice 2006, Cerdas y Montero 2016). Costa Rica es el principal exportador en América Central, con un valor de \$5,1 millones anuales, que beneficia cerca de 200 familias e agricultoras de las provincias de Limón y Cartago (Lavagni y Rodríguez 2009).

El sistema de cultivo actual, en suelo a campo abierto, ha presentado problemas de inocuidad, fitosanitarios y ambientales, que han puesto en riesgo la estabilidad del mercado en Estados Unidos. Entre las prácticas culturales convencionales, que afectan los rendimientos y rentabilidad, están la selección y tratamiento de semilla, la siembra al voleo, el uso intensivo de agroquímicos para el control de enfermedades, moluscos (babosas y caracoles), malezas y nematodos. Se estiman hasta 10 aplicaciones al año de Paraquat y Oxamyl, una por cada corta o cosecha. Consecuentemente, el culantro coyote es uno de los vegetales frescos que presenta 
mayor cantidad de residuos, que supera el límite máximo permitido (SFE 2014). Dichas prácticas de cultivo en suelo afectan los rendimientos, con un rango de 4 a 5 rollos de $200 \mathrm{~g}_{\text {por }} \mathrm{m}^{-2}$, frente a un potencial productivo de 20 rollos. $\mathrm{m}^{-2}$.

El rendimiento en culantro coyote es muy variable, ya que depende de diversos factores tales como el clima y el manejo del cultivo. En Costa Rica, el rendimiento promedio (40 t.ha-1. año $\left.{ }^{-1}\right)$ es inferior a los rendimientos reportados en Filipinas $\left(80\right.$ t.ha $\left.{ }^{-1} \cdot a_{n} o^{-1}\right)$ y similares a los reportados en Puerto Rico (46 t.ha ${ }^{-1} \cdot \mathrm{año}^{-1}$ ) (Morales et al. 2013).

Lo anterior, plantea la necesidad de mejorar el sistema de producción actual de culantro coyote, para reducir los efectos negativos sobre el ambiente, mejorar la inocuidad, la calidad, el rendimiento y dar valor agregado del producto final, para asegurar la sostenibilidad del sistema de producción. Una alternativa de mejora es la producción hidropónica en condiciones protegidas, el cual es un sistema de cultivo aislado del suelo, cuyos requerimientos hídricos y nutricionales se obtienen a través de una solución nutritiva. La agricultura protegida en hidroponía reduce los efectos de climas extremos, mejora la eficiencia de uso de agua, de los fertilizantes y del espacio, reduce enfermedades, así como aumenta la calidad y los rendimientos (Jara 2016), lo que permite estabilidad en la producción y acceso a mercados que opten por cultivos con prácticas de producción ventajosas como las mencionadas.

Adicional al sistema del cultivo, la protección con techos plásticos en época lluviosa podría reducir el lavado de fertilizantes, la incidencia de plagas y enfermedades y consecuentemente el uso de pesticidas, lo que genera productos más sanos para quien consume y menor contaminación ambiental (Rojas y Paniagua 2015). Estudios realizados han demostrado que, el sombreo en época, incrementa el rendimiento respecto al cultivo a pleno sol. Al respecto, Alvarado et al. (1999) plantean que bajo sombra las hojas de culantro son de mayor tamaño y de mejor calidad, en comparación al cultivo a pleno sol.
Además, se ha determinado que el uso de coberturas con sarán rojo presenta alta transmisividad de luz del espectro rojo entre 600-700 nm (OrenShamir et al. 2001), alta difusión de luz (Shahak et al. 2004), protege contra la radiación ultravioleta, evita la fotodegradación de los cloroplastos (Leite 2005) e incrementa la productividad en cultivos de hortalizas y ornamentales (Shahak et al. 2008).

Sin embargo, la principal limitación para la adopción de técnicas hidropónicas, bajo protección para el cultivo de culantro coyote hidropónico, es la falta de información acerca del manejo del agua y la nutrición. En absorción de nutrientes, únicamente se encontró un estudio de Bertsch (2003), para el cultivo de culantro coyote convencional en suelo y campo abierto. Las concentraciones de nutrientes habitualmente usadas en solución nutritiva para la producción hidropónica de cultivos de hoja son muy variables. Una de las más usadas por la mayoría de las personas hidrocultoras en Costa Rica para la producción de hojas, es la solución genérica del Instituto Nacional de Aprendizaje (Soto y Ramírez 2005), con concentraciones de nutrientes dentro de los habitualmente usados para la producción de vegetales de hoja a nivel internacional. Por tanto, al no haber soluciones nutritivas específicas para culantro coyote hidropónico, el uso de concentraciones bajas y altas, dentro de los de rangos habitualmente usados en cultivos hidropónicos de hojas, es una alternativa para explorar cómo se comporta la dinámica del agua y de nutrientes en un sistema cultivo hidropónico de culantro coyote.

La agricultura del futuro enfrenta el desafío de abastecer la creciente demanda de alimentos, producto del crecimiento de la población mundial (Tilman et al. 2002), frente al cambio climático y la potencial crisis global del agua (WWAP 2016). Elliott et al. (2014) señalan que la escasez de agua dulce para riego, como consecuencia del cambio climático, reduciría los rendimientos en los principales cultivos alimenticios a nivel global, tales como maíz, soja, trigo y arroz. Lo anterior, plantea la necesidad de realizar un 
uso eficiente del agua, mediante la innovación en nuevas tecnologías de producción.

El objetivo de este estudio fue evaluar el efecto del nivel de nutrición y de la protección del cultivo de culantro coyote hidropónico, sobre el rendimiento y parámetros de uso de agua: eficiencia de uso de agua (EUA), evapotranspiración de cultivo $\left(\mathrm{ET}_{\mathrm{c}}\right)$, drenaje y coeficientes de cultivo $\left(\mathrm{K}_{\mathrm{c}}\right)$.

\section{MATERIALES Y MÉTODOS}

El estudio fue realizado del 18 de abril al 7 de agosto del 2016, en la Estación Experimental Fabio Baudrit Moreno (EEFBM), ubicada en San José de Alajuela, Costa Rica $\left(10^{\circ} 01^{\prime} \mathrm{N}\right.$ y $84^{\circ} 16^{\circ}$ $\mathrm{O}, 840 \mathrm{msnm}$ ), con valores promedios anual de temperatura, humedad relativa y precipitación de $22^{\circ} \mathrm{C}, 78 \%$ y $1940 \mathrm{~mm}$, respectivamente.
Se utilizó un sistema de cultivo hidropónico en bancales de madera de $12 \mathrm{~m}$ de largo, $1 \mathrm{~m}$ de ancho y $0,1 \mathrm{~m}$ de profundidad, colocadas sobre una estructura de metal elevada a $0,8 \mathrm{~m}$ sobre la superficie del suelo. El contenedor se impermeabilizó con plástico negro de 7 micras para proteger la madera y evitar la pérdida de la solución nutritiva aplicada. Para evacuar el exceso de agua por lluvias, se colocó un orificio de drenaje en un extremo del contenedor a una altura aproximada de un $\mathrm{cm}$ del fondo de la cama.

Como sustrato se utilizó polvo de piedra, cuyas características físicas e hidráulicas (Tabla 1) fueron determinadas, según la norma europea UNE-EN-13041 (2007), en muestras sometidas a potenciales mátricos $\left(\psi_{\mathrm{m}}\right)$ de 1,0, 5,0 y $10 \mathrm{kPa}$ por medio de un equipo de caja de arena (Eikelkamp, mod. 08.01).

Tabla 1. Análisis físico del sustrato polvo de piedra utilizado en el cultivo de culantro coyote hidropónico.

\begin{tabular}{|c|c|c|c|}
\hline \multirow{2}{*}{ Parámetro } & \multicolumn{2}{|c|}{ Rangos óptimos ${ }^{1}$} & \multirow{2}{*}{ Polvo de piedra } \\
\hline & Máximo & Mínimo & \\
\hline Índice de grosor (\%) & & & 65,35 \\
\hline Densidad aparente $\left(\mathrm{g} \cdot \mathrm{ml}^{-1}\right)$ & 0,15 & $<0,15$ & 1,14 \\
\hline Densidad real (g.ml-1) & 1,0 & $<1,0$ & 2,4 \\
\hline Porosidad total $\left(\mathrm{cm} . \mathrm{cm}^{-3}\right)$ & - & $>85$ & 52,5 \\
\hline Fracción sólida $\left(\mathrm{cm} \cdot \mathrm{cm}^{-3}\right)$ & 0,15 & $<0,15$ & 47,5 \\
\hline Componentes totales $\left(\mathrm{cm} \cdot \mathrm{cm}^{-3}\right)$ & 100,0 & 100,0 & 100,0 \\
\hline \multicolumn{4}{|l|}{ Humedad volumétrica $\left(\mathrm{cm} \mathrm{cm}^{-3}\right)$} \\
\hline $1 \mathrm{kPa}$ (capacidad de contenedor) & 55,0 & 70,0 & 19,0 \\
\hline $5 \mathrm{kPa}$ & 30,0 & 40,0 & 14,4 \\
\hline $10 \mathrm{kPa}$ (Punto de marchitez) & 25,0 & 30,0 & 9,6 \\
\hline \multicolumn{4}{|l|}{ Capacidad de aireación $\left({\left.\mathrm{g} . \mathrm{cm}^{-3}\right)}^{-3}\right.$} \\
\hline $1 \mathrm{kPa}$ (capacidad de contenedor) & 30,0 & 15,0 & 33,0 \\
\hline $5 \mathrm{kPa}$ & 55,0 & 45,0 & 38,10 \\
\hline $10 \mathrm{kPa}$ (Punto de marchitez) & 60,0 & 55,0 & 42,9 \\
\hline \multicolumn{4}{|l|}{ Tipos de agua $\left({\left.\mathrm{g} . \mathrm{cm}^{-3}\right)}^{-3}\right.$} \\
\hline Agua fácilmente disponible & 20,0 & 30,0 & 5,10 \\
\hline Agua de reserva & 5,0 & 10,0 & 4,80 \\
\hline Agua total disponible & 25,0 & 40,0 & 9,90 \\
\hline Agua difícilmente disponible & 30,0 & 30,0 & 9,60 \\
\hline
\end{tabular}

\footnotetext{
${ }^{1}$ Adaptado de Ansorena (1994).
} 
Como semilla, se utilizó la variedad local cultivada por las personas agricultoras para exportación, que fue recolectada de diferentes fincas de la zona de producción de Tres Equis, Turrialba. La semilla fue limpiada, seleccionada y se realizaron pruebas de germinación, de las cuales se obtuvieron porcentajes germinación mínimos de $40 \%$ y máximos de $78 \%$.

La siembra se realizó por trasplante de plántulas de 60 días edad con aproximadamente 7 hojas verdaderas. La densidad de siembra utilizada fue de 7 x $7 \mathrm{~cm}$ (49 plantas. $\left.\mathrm{m}^{-2}\right)$. Una semana antes del trasplante, se desinfectó el sustrato con tiocianatometiltio-benzotiazol (TCMTB) y el día previo al trasplante se aplicó Trichoderma y extracto de algas a las plántulas.

Se utilizó un sistema de riego por gravedad, conformado 2 por tanques de $2500 \mathrm{~L}$, tubería primaria de polietileno (1") y cintas con goteros incorporados cada $20 \mathrm{~cm}\left(0,8 \mathrm{~L}^{-1} \mathrm{~h}^{-1}\right)$, manómetros (0-80 PSI), reguladores de presión (20 PSI) y válvulas solenoides. Las cintas de goteo fueron distribuidas a razón de una por cada hilera de cultivo separadas a $7 \mathrm{~cm}$. Durante el ciclo de cultivo, se determinó el coeficiente de uniformidad del sistema de riego (CU: 96 - 98\%) y el caudal promedio de los goteros según Christiansen (1942).

El manejo fitosanitario del cultivo se realizó de acuerdo con un programa preventivo de productos biológicos y orgánicos como Beauveria bassiana, Metarhizium y con un repelente a base de extracto de Neem. El manejo preventivo de plagas y enfermedades se complementó con monitoreos semanales para la toma de decisiones de aplicación.

El estudio comprendió 2 etapas de producción consecutivas, en un mismo ciclo de cultivo, denominadas en adelante etapa I y etapa II. La etapa I coincidió con la época seca y el cultivo estuvo protegido con sarán rojo (30\% de sombra) desde el trasplante hasta la primera cosecha. La etapa II de producción comprendió la época lluviosa, donde el cultivo fue protegido con techo plástico $(50 \mu$ de grosor $)$ desde el primer corte hasta la segunda cosecha. En ambas épocas se aplicaron 4 tratamientos que combinaron 2 condiciones ambientales: un cultivo protegido $(\mathrm{CP})$ y otro sin protección (SP), con 2 niveles de nutrición: alta (NA) y baja concentración de nutrientes (NB) en solución nutritiva (Tabla 2).

Tabla 2. Descripción tratamientos utilizados en el experimento, en etapas I y II del cultivo de culantro coyote hidropónico en época seca y lluviosa.

\begin{tabular}{|c|c|c|c|}
\hline Época y fecha & $\begin{array}{l}\text { Tratamiento } \\
\text { (Siglas) }\end{array}$ & $\begin{array}{l}\text { Condición } \\
\text { de luz }\end{array}$ & Nutrición \\
\hline \multirow{4}{*}{$\begin{array}{l}\text { Transición época seca-lluviosa, } \\
\text { del 18/04 al 12/06/2016 }\end{array}$} & Con protección/nutrición alta (CA) & $30 \%$ sombra & Alta \\
\hline & Con protección/nutrición baja (CB) & $30 \%$ sombra & Baja \\
\hline & Sin protección /nutrición alta (SA) & Pleno sol & Alta \\
\hline & Sin protección /nutrición baja (SB) & Pleno sol & Baja \\
\hline \multirow{4}{*}{ Época lluviosa, del 13/06 al 07/08/2016 } & Con protección/nutrición alta (CA) & Techo plástico & Alta \\
\hline & Con protección/nutrición baja (CB) & Techo plástico & Baja \\
\hline & Sin protección/nutrición alta (SA) & Plena lluvia & Alta \\
\hline & Sin protección/nutrición baja (SB) & Plena lluvia & Baja \\
\hline
\end{tabular}


Para la instalación de la protección con sarán o techo plástico, se utilizaron arcos de varillas de metal $(12,5 \mathrm{~mm})$ de $1,2 \mathrm{~m}$ de alto y $1,5 \mathrm{~m}$ de ancho, colocados sobre las camas de cultivo y espaciados cada $\mathrm{m}$.
Las concentraciones de nutrientes en los tratamientos, de baja (NB) y alta concentración (NA) (Tabla 3), fueron definidas considerando los rangos utilizados para la producción hidropónica de vegetales de hojas a nivel nacional e internacional (Urrestarazu 2015, Resh 2001, Soto y Ramírez 2005, Rodríguez et al. 2001).

Tabla 3. Concentración de nutrientes en las soluciones nutritivas utilizadas en los tratamientos con nivel de nutrición baja (NB) y alta (NA).

\begin{tabular}{|c|c|c|c|c|c|c|c|c|c|c|c|c|c|}
\hline \multirow{2}{*}{ Tratamiento } & \multicolumn{12}{|c|}{ Concentración (mg.L $\left.\mathrm{L}^{-1}\right)$} & \multirow{2}{*}{$\begin{array}{c}\mathrm{CE} \\
\mathrm{uS} \cdot \mathrm{c}^{\mathrm{m}-1}\end{array}$} \\
\hline & $\mathrm{N}$ & $\mathrm{P}$ & K & $\mathrm{Mg}$ & $\mathrm{S}$ & $\mathrm{Ca}$ & $\mathrm{Fe}$ & $\mathrm{Cu}$ & $\mathrm{Mn}$ & $\mathrm{Zn}$ & B & Mo & \\
\hline Baja (NB) & 75 & 17 & 100 & 18 & 32 & 81 & 0,4 & 0,1 & 0,2 & 0,1 & 0,2 & 0,04 & 0,8 \\
\hline Alta (NA) & 225 & 52 & 300 & 53 & 97 & 225 & 2,0 & 0,5 & 1,0 & 0,5 & 1,0 & 0,20 & 2,3 \\
\hline
\end{tabular}

En la preparación de la solución nutritiva se utilizó fosfato monopotásico, nitrato de potasio, sulfato de potasio, sulfato de magnesio, nitrato de calcio, cloruro de calcio, ácido nítrico, elementos menores (Fetrilón comb-1), ácido bórico y quelatos de hierro y de manganeso (EDTAFe, EDTA-Mn).

Se utilizó un diseño de bloques completos al azar en parcelas divididas con arreglo bifactorial $2 \times 2$, con 4 tratamientos que combinaron las 2 condiciones de protección con los 2 niveles de nutrición. La parcela principal correspondió a la condición de protección y la subparcela al nivel de nutrición. Se utilizaron 5 repeticiones por tratamiento, para un total de 20 unidades experimentales. El área de la unidad de muestreo semanal, fue de $3 \mathrm{~m}^{2}$ para variables de crecimiento y de $1 \mathrm{~m}^{2}$ para variables de riego y de rendimiento.

La demanda hídrica del cultivo (L.m ${ }^{-2}$. $\left.\mathrm{dia}^{-1}\right)$ fue estimada al considerar i- un porcentaje de agotamiento (15\%) del agua total disponible en el sustrato (Tabla 1), ii- el volumen de sustrato $\left(\mathrm{L}^{\mathrm{m}} \mathrm{m}^{-2}\right)$ y iii- un porcentaje de drenaje preestablecido (+-20\%). Una vez determinado el requerimiento de agua $\left(\mathrm{L} \cdot \mathrm{m}^{-2} \cdot \mathrm{dia}^{-1}\right)$, se calculó el tiempo de riego a partir del número de goteros por $\mathrm{m}^{-2} \mathrm{y}$ del caudal promedio por gotero obtenido según CU. Dicho requerimiento $\left(\mathrm{L} \cdot \mathrm{m}^{-2} \cdot \mathrm{dia}^{-1}\right)$ fue distribuido en riegos con horarios cortos y frecuentes, según tratamientos de nutrición baja y alta. La frecuencia de riego fue ajustada diariamente en función del porcentaje de drenaje obtenido, el cual dependió de las condiciones climáticas y de crecimiento del cultivo.

En cada etapa del experimento, se caracterizó el microclima para cada condición ambiental del cultivo (CP y SP), con un sensor de temperatura y humedad relativa (HOBO Mod. Pro V2). Los datos fueron almacenados en un registrador de datos o Data logger (Campbell Scientific. Mod. CR1000) y se terminaron los promedios temperatura y humedad relativa de valores máximos, mínimos y promedios del ciclo de cultivo (Tabla 4). La precipitación $(\mathrm{mm})$ fue medida por 4 pluviómetros (Decagon Devices Mod. ECRN-50), 2 en los tratamientos CP y 2 en los cultivos SP. La radiación solar global y la velocidad de viento (V_V) fueron obtenidos de la Estación Meteorológica del Instituto Meteorológico Nacional, ubicada a $100 \mathrm{~m}$ del cultivo experimental. La radiación solar dentro de los cultivos $\mathrm{CP}$ se estimó a partir de un coeficiente de transmisividad de luz, obtenido como el cociente entre los valores de radiación PAR en los cultivos CP y en SP, medidos con barras lineales de radiación PAR (APOGEE Mod. LQS100-20). En ambas etapas 
de cultivo de culantro coyote, se determinó la integral de radiación solar diaria (DLI: mol.m ${ }^{-2}$. día $\left.\mathrm{a}^{-1}\right)$ y el déficit de presión de vapor $(\mathrm{kPa})$ en los tratamientos con y sin protección.

Tabla 4. Valores promedios de temperatura y humedad relativa máximas, mínimas y promedios, durante la etapa I en época seca y etapa II en época lluviosa, de un cultivo de culantro coyote hidropónico, bajo condición protegida (CP) y sin protección (SP). Alajuela, Costa Rica. 2016.

\begin{tabular}{|c|c|c|c|c|c|c|c|c|c|c|}
\hline \multirow{2}{*}{ Etapa } & \multirow{2}{*}{ Protección } & \multicolumn{3}{|c|}{ Temperatura $\left({ }^{\circ} \mathrm{C}\right)$} & \multicolumn{3}{|c|}{ Humedad relativa (\%) } & \multicolumn{3}{|c|}{ Humedad relativa (\%) } \\
\hline & & Máx. & Mín. & Prom. & Máx. & Mín. & Prom. & Máx. & Mín. & Prom. \\
\hline \multirow{2}{*}{1} & SP & 31,96 & 19,49 & 24,59 & 98,81 & 61,90 & 86,59 & 3,83 & 1,54 & 2,69 \\
\hline & CP & 32,56 & 19,58 & 24,82 & 98,61 & 60,49 & 85,95 & 1,98 & 0,03 & 1,0 \\
\hline \multirow{2}{*}{2} & SP & 31,72 & 18,70 & 23,92 & 100,0 & 63,26 & 88,81 & 3,82 & 1,47 & 2,65 \\
\hline & $\mathrm{CP}$ & 31,42 & 18,96 & 23,90 & 99,96 & 63,62 & 88,61 & 1,70 & 0,0 & 0,85 \\
\hline
\end{tabular}

A continuación, se describen las condiciones ambientales que caracterizaron al cultivo, aportes de agua, la humedad volumétrica, salinidad en el sustrato e incluso el clima.

Los valores de DLI fueron superiores en condiciones SP, mientras que la V_V fue similar en ambas condiciones, debido a que fue un sistema abierto sin paredes laterales. Los promedios globales de DLI, en etapa I, fueron 34,2 y $21,2\left(\mathrm{~mol} . \mathrm{m}^{-2} \cdot\right.$ día $\left.^{-1}\right)$ para los tratamientos SP y $\mathrm{CP}$, respectivamente (Figura 1); mientras que en etapa II, se obtuvieron valores de 31,8 en SP y 20,0 (mol.m $\left.\mathrm{m}^{-2} \cdot \mathrm{dí}^{-1}\right)$ en CP.

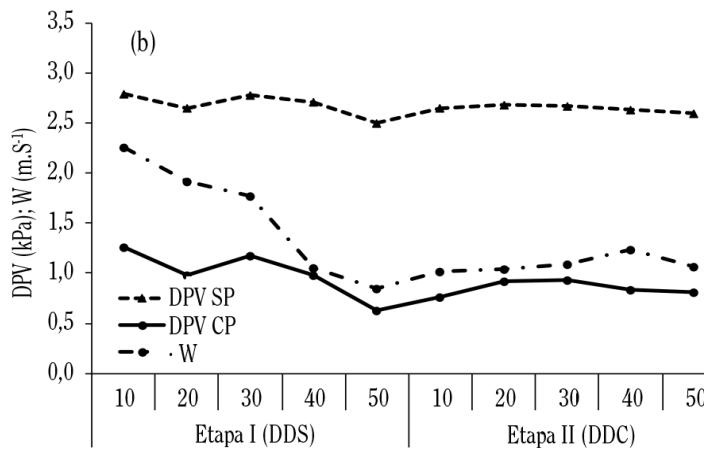

Figura 1. Valores promedios de (a) integral de radiación solar diaria (DLI), (b) déficit de presión de vapor (DPV) y velocidad de viento (VV), durante la etapa I en época seca y etapa II en época lluviosa, de un cultivo de culantro coyote hidropónico, bajo condición protegida (CP) y sin protección (SP). Las siglas DDT y DDC indican días después de trasplante y después de cosecha, respectivamente. Alajuela, Costa Rica. 2016.

Los valores promedios de temperatura y humedad relativa máximas, mínimas y promedios, durante el ciclo del experimento (Tabla 4), fueron muy similares, debido a que fue un sistema abierto verticalmente.
El volumen de agua aportada $\left(\mathrm{L} . \mathrm{m}^{-2}\right)$, incluyendo riego y precipitación, varió dependiendo del tipo de protección y de la época climática (Figura 2). En la etapa I de cultivo en época seca, el volumen de riego (R) fue similar 
en ambas condiciones de protección (Figura 2a), la precipitación (PP) fue un $15 \%$ superior en el cultivo SP (Figura 2a) y el aporte total de agua $(\mathrm{R}+\mathrm{PP})$ fue un $10 \%$ superior en el cultivo SP.
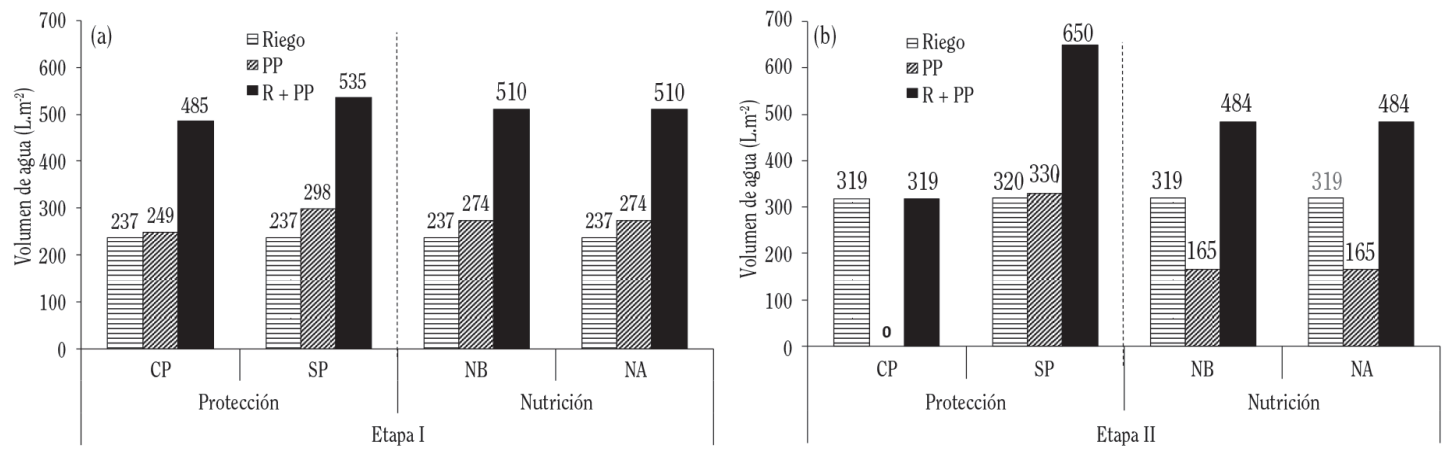

Figura 2. Volúmenes de (a) riego aplicado (R), (b) precipitación (PP) y (c) riego + precipitación (R+PP) durante (a) etapa I en época seca y (b) etapa II en época lluviosa, de un cultivo de culantro coyote hidropónico, bajo protección (CP) y sin protección (SP).

Alajuela, Costa Rica. 2016.

En la etapa II en época lluviosa, el riego aportado en ambas condiciones de cultivo fue similar, sin embargo, el aporte total de agua en el cultivo SP fue $103 \%$ superior al cultivo protegido con plástico $(\mathrm{CP})$, ya que la protección evitó el aporte de agua de lluvia en CP (Figura 2b).
La evolución del contenido de humedad volumétrica, en el sustrato en etapas I y II, fue similar en ambas condiciones de protección (Figura 3a) y de nutrición (Figura 3b), con un valor promedio de $0,37 \mathrm{~cm}^{3} \cdot \mathrm{cm}^{-3}$, independiente de la etapa y de los factores evaluados.
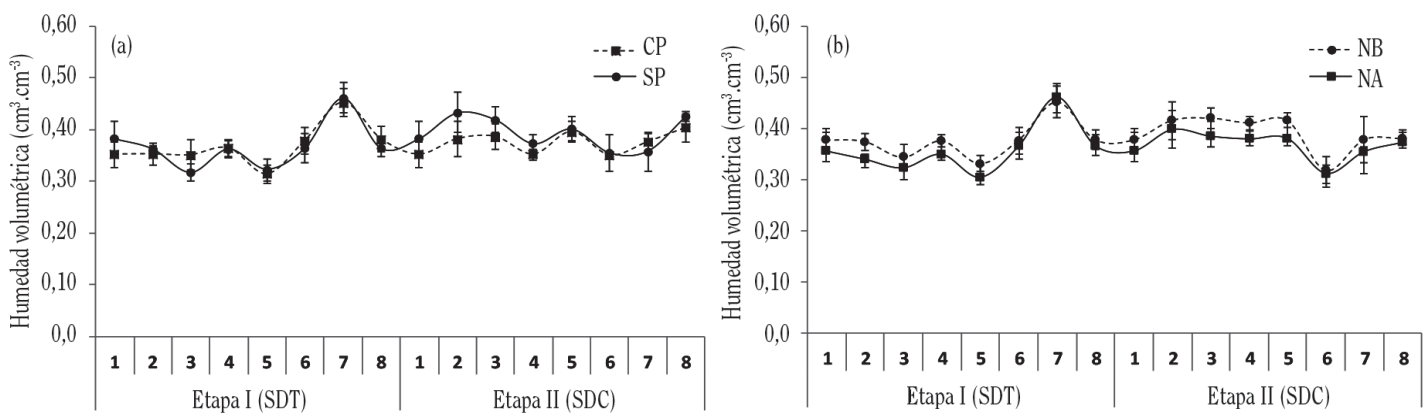

Figura 3. Valores promedio de humedad volumétrica en el sustrato, durante la etapa I en época seca y etapa II en época lluviosa, de un cultivo de culantro coyote hidropónico, bajo condiciones (a) con (CP) y sin (SP) protección y (b) con nutrición baja (NB) y alta (NA). Las siglas SDT y SDC indican la semana después de trasplante y después de cosecha, respectivamente. Alajuela, Costa Rica. 2016.

En ambas etapas de cultivo, la conductividad eléctrica (CE) y el $\mathrm{pH}$ de cada tratamiento fueron medidas diariamente antes de iniciar los riegos mediante un pHmetro-conductivímetro (HANNA Mod. HI98129). Para ello, se recolectaron muestras del agua drenada el día anterior, 
desde un recipiente que recolectaba el agua drenada. Los niveles de CE en el drenaje fueron superiores cuando se consideró el nivel de nutrición (Figura 4). Según la condición de protección (Figura 4a), la CE en el drenaje fue similar en etapa I, mientras que en etapa II fue mayor en el cultivo $\mathrm{CP}$, ya que alcanzó valores máximos de 2 uS.cm ${ }^{-1}$. De acuerdo con el nivel de nutrición (Figura 4b), en ambas etapas de cultivo los valores de $\mathrm{CE}$ en el drenaje fueron superiores con nivel de nutrición alto, donde alcanzó valores cercanos a 3 uS.cm ${ }^{-1}$.
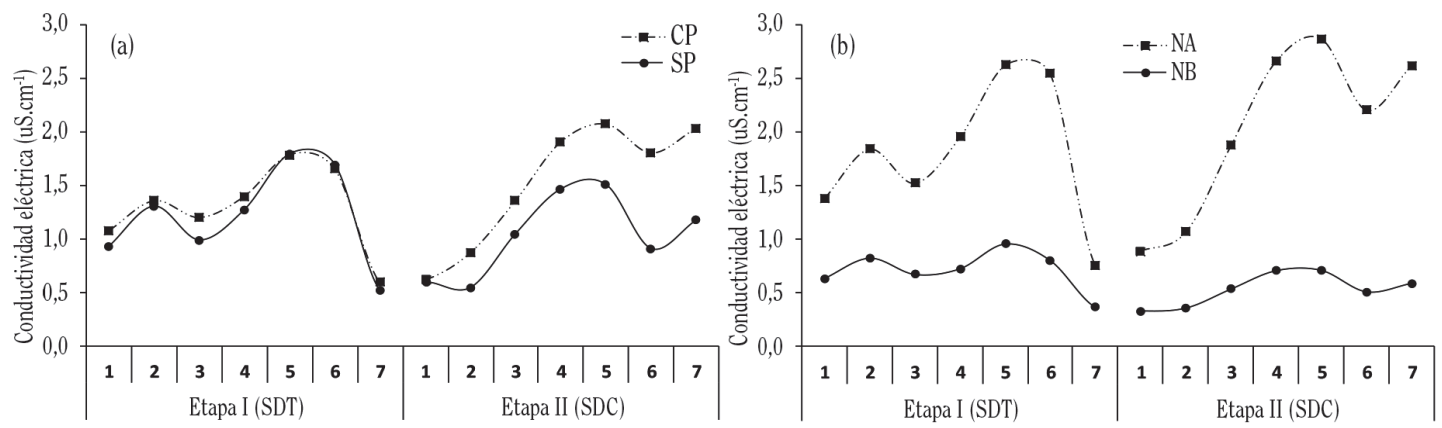

Figura 4. Evolución de la conductividad eléctrica (CE) en solución drenada, durante la etapa I en época seca y etapa II en época lluviosa, de un cultivo de culantro coyote hidropónico, (a) bajo condiciones con (CP) y (b) sin (SP) protección y con nutrición baja (NB) y alta (NA). Las siglas SDT y SDC indican la semana después de trasplante y después de cosecha, respectivamente.

Alajuela, Costa Rica. 2016.

Variables de respuesta. En cada etapa de cultivo se evaluaron parámetros de uso de agua, que incluyó el rendimiento fresco y parámetros de uso de agua del cultivo: eficiencia de uso de agua (EUA), evapotranspiración del cultivo $\left(\mathrm{ET}_{\mathrm{c}}\right)$, drenaje y coeficientes de cultivo $\left(\mathrm{K}_{\mathrm{c}}\right)$. La $\mathrm{ET}_{\mathrm{c}}$ se determinó para periodos de 10 días, con el método de balance de agua, a partir de los aportes de agua de riego (R) y precipitación (P), los volúmenes de drenaje (D) y de la diferencia entre el contenido de humedad volumétrica del sustrato $\left(\Delta \theta=\theta_{\mathrm{i}}-\theta_{\mathrm{f}}\right)$ al inicio $\left(\theta_{\mathrm{i}}\right)$ y al final $\left(\theta_{\mathrm{f}}\right) \mathrm{de}$ cada periodo, según la ecuación $\mathrm{ET}_{\mathrm{c}}=\mathrm{R}+\mathrm{P}-\mathrm{D}$ $\pm \Delta \theta$ (Soto 2018). Adicionalmente, se estimó el promedio de $\mathrm{ET}_{\mathrm{c}}$ diario por periodo decenario y la $\mathrm{ET}_{\mathrm{c}}$ total acumulada durante cada época del ciclo de cultivo.

El volumen drenado y la $\theta$ se midieron diariamente a las 6 a.m. antes del inicio del primer riego. El contenido de $\theta\left(\% \mathrm{v} \mathrm{v}^{-1}\right)$ se midió con un sensor de capacitancia "TDR" (Time Domain Reflectometry, Decagon Devices mod. GS3).

La evapotranspiración de referencia $\left(\mathrm{ET}_{\mathrm{o}}\right)$ fue estimada utilizando el método PenmanMonteith FAO-56 (Allen et al. 2006).

$$
\frac{E T_{o}=\left[\frac{1}{\lambda}\right] \Delta\left(R_{n}-\mathrm{G}\right)+\gamma \frac{900}{\mathrm{~T}+273} u_{2}+\left(e_{s}-e_{a}\right)}{\Delta+\mathrm{Y}\left(1+0,34 \mathrm{u}_{2}\right)}
$$


Donde: $\mathrm{ET}_{\mathrm{o}}$ es la evapotranspiración de referencia (mm.día- $\left.{ }^{1}\right), \lambda$ es el calor latente de vaporización para convertir el componente de radiación en la $\mathrm{ET}_{0}$ de $\mathrm{MJ} . \mathrm{m}^{-2}$.día ${ }^{-1}$ a mm.día- ${ }^{1}$, $\mathrm{R}_{\mathrm{n}}$ es la radiación neta $\left(M J \cdot \mathrm{m}^{-2} \cdot \mathrm{dí}^{-1}\right), \mathrm{G}$ es el flujo de calor en el suelo $\left(\mathrm{MJ}_{\mathrm{H}} \mathrm{m}^{-2} \cdot \mathrm{dí}^{-1}\right)$, T es la temperatura promedio del aire $\left({ }^{\circ} \mathrm{C}\right), \Delta$ es la pendiente de la curva de presión a saturación $\left(\mathrm{kPa} .{ }^{\circ} \mathrm{C}^{-1}\right), \gamma$ es la constante psicométrica $\left(\mathrm{kPa} .{ }^{\circ} \mathrm{C}^{-1}\right), \mathrm{e}_{\mathrm{s}}$ la presión de saturación del vapor $(\mathrm{kPa}), \mathrm{e}_{\mathrm{a}}$ es la presión de vapor promedio diaria ( $\mathrm{kPa}) \mathrm{y} \mathrm{u}_{2}$ es la velocidad promedio del viento a $2 \mathrm{~m}$ de altura $\left(\mathrm{m} \cdot \mathrm{s}^{-1}\right)$.

Los coeficientes de cultivo $\left(\mathrm{K}_{\mathrm{c}}\right)$ se estimaron como promedios diarios de cada semana de cultivo, como el cociente entre $\mathrm{ET}_{\mathrm{c}} \mathrm{y} \mathrm{ET}_{\mathrm{o}}$ (Allen et al. 2006).

$$
K_{c}=\frac{E T_{c}}{E T_{o}}
$$

La cosecha se realizó a los 50 días después del trasplante (DDT) en etapa I y 50 días después de primera cosecha (DDC) en etapa II. El rendimiento fresco se obtuvo de 25 plantas (macolla) cosechadas en la parte central de cada
UE, dejando las hileras externas como borde. A partir del peso medio por planta (g), se estimó el rendimiento fresco $\left(\mathrm{Kg} . \mathrm{m}^{-2}\right)$ para la densidad de siembra utilizada (49 plantas. $\mathrm{m}^{-2}$ ). La eficiencia de uso de agua (EUA, $\mathrm{kg} \cdot \mathrm{m}^{-3}$ ) se estimó como el cociente entre rendimiento fresco $\left(\mathrm{kg} \cdot \mathrm{m}^{-2}\right)$ y la $\operatorname{ET}_{\mathrm{c}}\left(\mathrm{m}^{3} \cdot \mathrm{m}^{-2}\right)$.

Análisis estadístico. Los datos obtenidos, que cumplieron los supuestos de normalidad y homocedasticidad, fueron sometidos a un análisis de varianza (ANDEVA) para determinar si hubo efecto de la interacción entre los factores de protección y nutrición, o efectos independientes de ambos factores, usando el software estadístico InfoStat versión 2016. Cuando se presentó interacción $(p<0,05)$, se realizó un análisis en dirección y magnitud de los efectos simples de ambos factores, mientras que cuando no hubo efecto de la interacción ( $\mathrm{p}>0,05)$, se analizó el efecto principal del factor o de ambos factores, que fueron estadísticamente significativos $(p<0,05)$. Para el ANDEVA, se utilizó un modelo estadístico lineal para bloques completos al azar en parcelas divididas según Di Rienzo et al. (2012).

$$
Y_{\mathrm{ijk}}=\mu+\tau_{\mathrm{i}}+\beta_{\mathrm{j}}+(\tau \beta)_{\mathrm{ij}}+\gamma \mathrm{ijk}+(\tau \gamma)_{i k}+\varepsilon_{\mathrm{ijk}}
$$

Dónde:

$\mathrm{Y}_{\mathrm{ijk}}=$ representa la observación en el k-ésimo nivel del factor aplicado a la subparcela, de la i-ésima parcela principal en el j-ésimo bloque.

$\mu=$ media general.

$\mathrm{T}_{\mathrm{i}}=$ el efecto del i-ésimo nivel del factor aplicado a la parcela principal.

$\beta_{\mathrm{j}}=$ el j-ésimo bloque.

$(\mathrm{G} \beta)_{\mathrm{ij}}=$ el error experimental de las parcelas principales que se simboliza como la interacción entre el factor principal y los bloques. $\gamma_{\mathrm{ijk}}=$ el efecto del k-ésimo nivel del factor asociado a la subparcela dentro de la i-ésima parcela principal del j-ésimo bloque.

$(\tau \gamma)_{\mathrm{ik}}=$ la interacción del factor principal con el factor aplicado a las subparcelas y

$\mathrm{E}_{\mathrm{ijk}}=$ el error experimental a nivel de subparcelas.

\section{RESULTADOS}

En el presente estudio, no hubo interacción entre los factores protección y nutrición $(\mathrm{p}>0,05)$ para todas las variables evaluadas, incluyendo la eficiencia de uso de agua (EUA), la evapotranspiración de cultivo $\left(\mathrm{ET}_{\mathrm{c}}\right)$, el drenaje, 
los coeficientes de cultivo $\left(\mathrm{K}_{\mathrm{c}}\right)$ y el rendimiento fresco. Sin embargo, se presentó efecto individual de dichos factores $(\mathrm{p}<0,05)$, según la variable evaluada.

Evapotranspiración y coeficientes de cultivo. En la Figura 5, se presentan los promedios diarios de evapotranspiración de referencia $\left(\mathrm{ET}_{\mathrm{o}}\right)$ y del cultivo $\left(\mathrm{ET}_{\mathrm{c}}\right)$ utilizados para estimar los coeficientes de cultivo $\left(\mathrm{K}_{\mathrm{c}}\right)$, para periodos de 10 días en las etapas I y II, donde se observa que el cultivo SP evapotranspiró mayor volumen de agua que el CP $(\mathrm{p}<0,05)$. En etapa I, en el cultivo $\mathrm{CP}$, la $\mathrm{ET}_{\mathrm{c}}$ diaria varió entre 2,0 y $3,8 \mathrm{~L} \cdot \mathrm{m}^{-2}$; mientras que en el cultivo SP alcanzó valores más altos (Figura 5a) que oscilaron entre 3,9 y $5,1 \mathrm{~L} . \mathrm{m}^{-2}$. En etapa II (Figura 5b) la $\mathrm{ET}_{\mathrm{c}}$ fluctuó entre 0,6 y $4,1 \mathrm{~L} \cdot \mathrm{m}^{-2}$ en el cultivo CP, y entre 2,9 y $5,4 \mathrm{~L} \cdot \mathrm{m}^{-2}$ en condiciones SP.
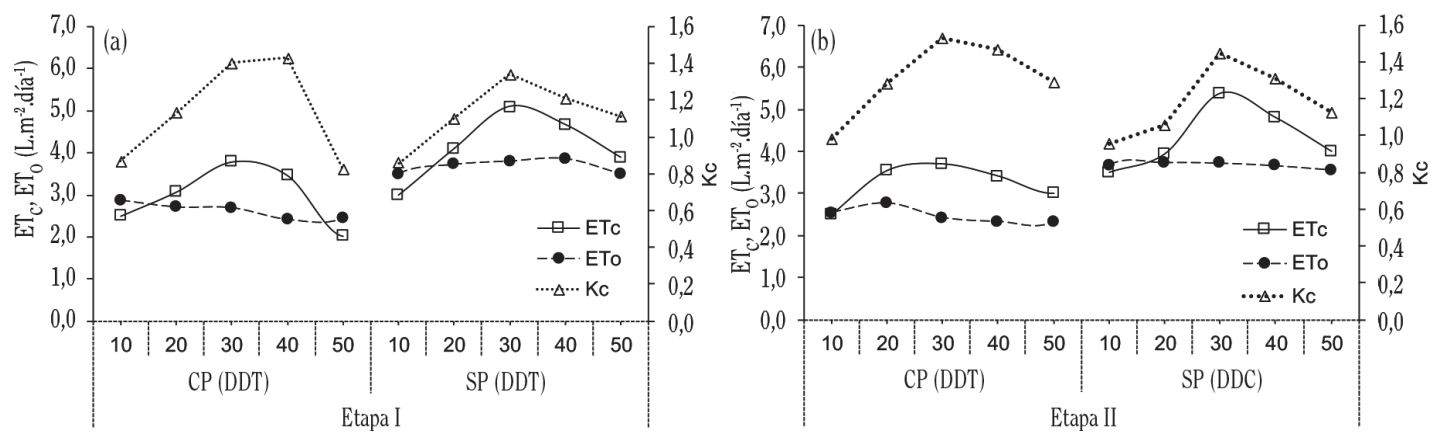

Figura 5. Promedios diarios de evapotranspiración del cultivo $\left(\mathrm{ET}_{\mathrm{c}}\right)$ y de referencia $\left(\mathrm{ET}_{\mathrm{o}}\right)$, y coeficientes de cultivo $\left(\mathrm{K}_{\mathrm{c}}\right)$ para periodos de 10 días, durante (a) etapa I, después de trasplante, en época seca y (b) etapa II, después de la primera cosecha, en época lluviosa, de un cultivo de culantro coyote hidropónico, bajo condición protegida (CP) y sin protección (SP), con baja (NB) y alta (NA) concentración de nutrientes. Las siglas DDT y DDC indican días después de trasplante y después de cosecha, respectivamente.

Alajuela, Costa Rica. 2016

En ambas etapas de cultivo (Figura 5), la $\mathrm{ET}_{\mathrm{o}}$ mostró un comportamiento similar, donde el cultivo SP fue en promedio un 38\% superior al ciclo $\mathrm{CP}$, con valores promedio de $2,55 \mathrm{~L} . \mathrm{m}^{-2}$ bajo CP y de 3,75 L.m ${ }^{-2}$ en el cultivo SP.

Los valores de $\mathrm{K}_{\mathrm{c}}$ en ambas etapas del cultivo de culantro coyote hidropónico, no fueron afectados por la interacción, ni individualmente, por la condición de protección y el nivel de nutrición $(\mathrm{p}>0,05)$. Por tanto, debido a la importancia de disponer de datos de $\mathrm{K}_{\mathrm{c}}$ para estimar la $\mathrm{ET}_{\mathrm{c}}$ en cultivo de culantro coyote hidropónico, en la Figura 5 se muestra el comportamiento de los $\mathrm{K}_{\mathrm{c}}$ bajo protección y sin protección en ambas etapas de cultivo, producto de la proporción entre promedios diarios de $\mathrm{ET}_{\mathrm{c}} / \mathrm{ET}_{\mathrm{o}}$ para periodos de 10 días $\left(\mathrm{ET}_{\mathrm{c}} / \mathrm{ET}_{\mathrm{o}}=\mathrm{K}_{\mathrm{c}}\right)$. A pesar de que los valores de $\mathrm{ET}_{\mathrm{c}}$ y $\mathrm{ET}_{\mathrm{o}}$ fueron superiores en el cultivo $\mathrm{SP}$, la proporción $\mathrm{ET}_{\mathrm{c}} / \mathrm{ET}_{\mathrm{o}}$ se mantuvo constante en ambas condiciones, por lo cual no hubo efecto estadísticamente significativo sobre $\operatorname{los} \mathrm{K}_{\mathrm{c}}$ de culantro coyote en ambas condiciones ambientales. Una situación similar se presentó al evaluar el efecto de la nutrición (datos no presentados).

Por tanto, independientemente de la condición de protección y del nivel de nutrición, los valores promedios de $\mathrm{K}_{\mathrm{c}}$ evolucionaron desde 0,9 en los primeros 10 días de cultivo hasta alcanzar valores máximos de 1,4 a los 30 días y disminuyendo a 1,1 a los 50 días. 
Evapotranspiración, rendimiento y eficiencia de uso de agua. El promedio, de $\mathrm{ET}_{\mathrm{c}}$ total bajo sombreo con sarán en etapa I (Figura 6a) y bajo techo plástico en etapa II (Figura 6b), no varió conforme incrementó el nivel de nutrición, debido a la ausencia de interacción

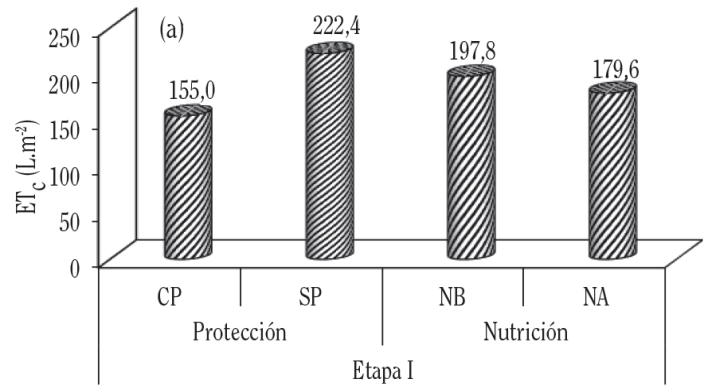

$(\mathrm{p}>0,05)$; mientras que hubo efecto individual únicamente del factor protección $(\mathrm{p}<0,05)$. En ambas épocas de cultivo, la $\mathrm{ET}_{\mathrm{c}}$ total disminuyó con magnitud similar, con un promedio de $43,5 \%$ al cambiar de un cultivo CP al cultivo SP (Figura 6a, 6b).

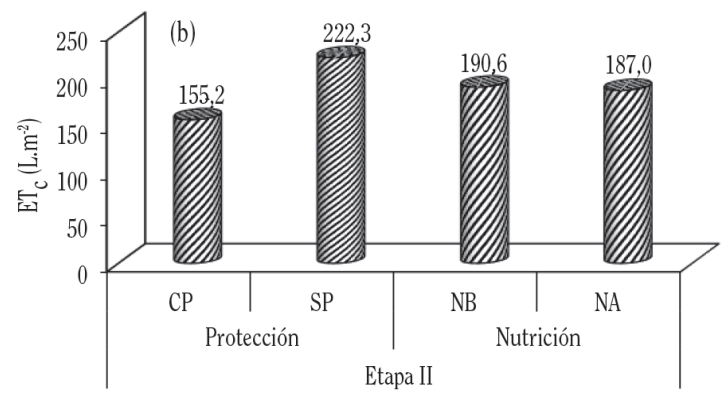

Figura 6. Evapotranspiración total del cultivo $\left(\mathrm{ET}_{\mathrm{c}}\right)$ durante (a) etapa I, después de trasplante, en época seca y (b) etapa II, después de la primera cosecha, en época lluviosa, en un cultivo de culantro coyote hidropónico, bajo condición protegida (CP) y sin protección (SP), con baja (NB) y alta (NA) concentración de nutrientes.

Alajuela. Costa Rica. 2016.

El rendimiento, en las etapas I (Figura 7a) y II (Figura $7 b)$ de cultivo, fue afectado $(\mathrm{p}<0,05)$ únicamente por el nivel de nutrición, sin interacción entre factores $(\mathrm{p}>0,05)$. En etapa $\mathrm{I}$, el rendimiento incrementó un $50 \%$, al pasar de bajo $\left(2,4 \mathrm{~kg} \cdot \mathrm{m}^{-2}\right)$ a alto nivel de nutrición $\left(3,7 \mathrm{~kg} \cdot \mathrm{m}^{-2}\right)$, Este cultivo habitualmente se comercializa en rollos de $200 \mathrm{~g}$, con lo cual, dicho rendimiento fue equivalente a 12 y 18 rollos $\mathrm{m}^{2}$ para los tratamientos con NB y NA, respectivamente. En etapa II (Figura 7b), el rendimiento fresco incremento un $44 \%$ al cambiar de NB $\left(5,6 \mathrm{~kg} . \mathrm{m}^{-2}\right.$ $=28$ rollos. $\left.\mathrm{m}^{-2}\right)$ a NA $\left(8,0 \mathrm{~kg} \cdot \mathrm{m}^{-2}=40\right.$ rollos $\left.\cdot \mathrm{m}^{-2}\right)$, equivalente a una diferencia de 12,2 rollos. $\mathrm{m}^{-2}$.
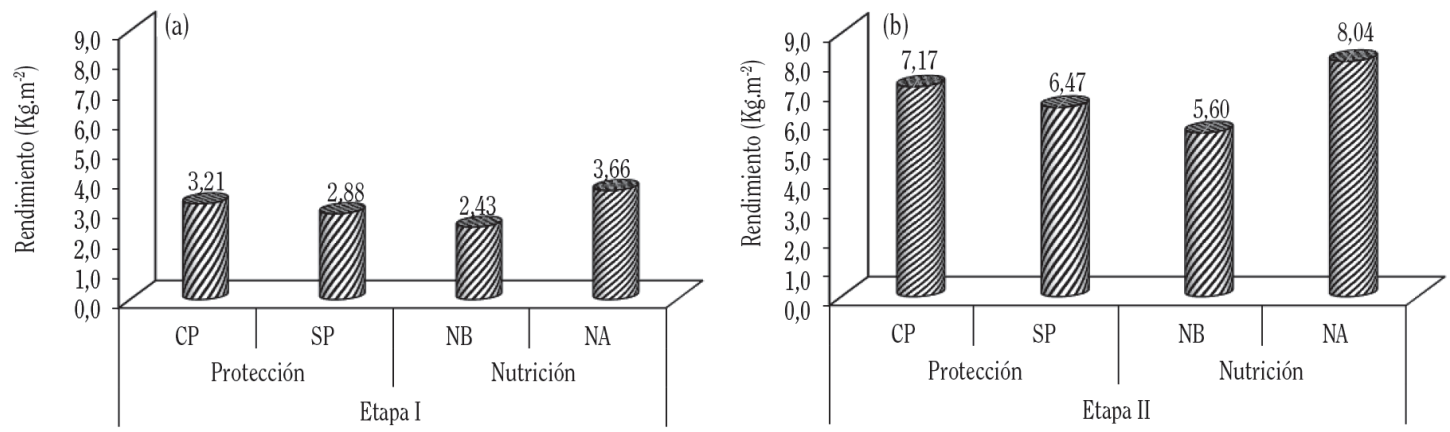

Figura 7. Rendimiento fresco durante (a) etapa I, después de trasplante, en época seca y (b) etapa II, después de la primera cosecha, en época lluviosa, de un cultivo de culantro coyote hidropónico, bajo condición protegida (CP) y sin protección (SP), con baja (NB) y alta (NA) concentración de nutrientes. Alajuela. Costa Rica. 2016. 
En la Figura 8 se presenta la evolución de la EUA en las etapas I (Figura 9a) y
II (Figura 9b) de cultivo de culantro coyote hidropónico.
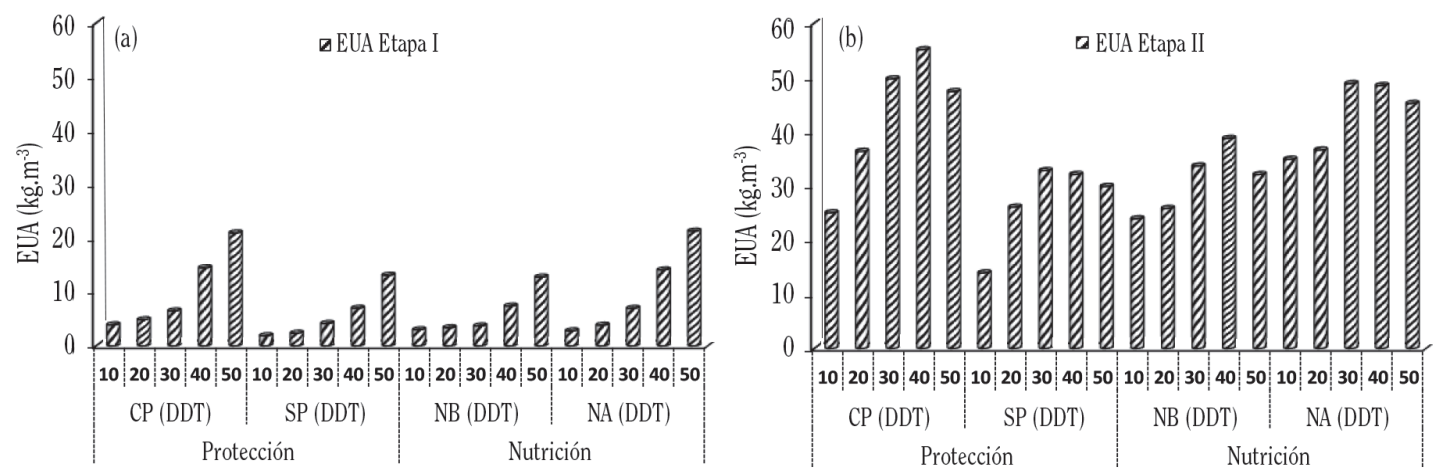

Figura 8. Evolución de la eficiencia de uso de agua (EUA) durante (a) etapa I, después de trasplante, en época seca y (b) etapa II, después de la primera cosecha, en época lluviosa, en un cultivo de culantro coyote hidropónico, bajo condición protegida $(\mathrm{CP})$ y sin protección (SP), con baja (NB) y alta (NA) concentración de nutrientes. Las siglas DDT y DDC indican días después de trasplante y después de cosecha, respectivamente.

Alajuela. Costa Rica. 2016.
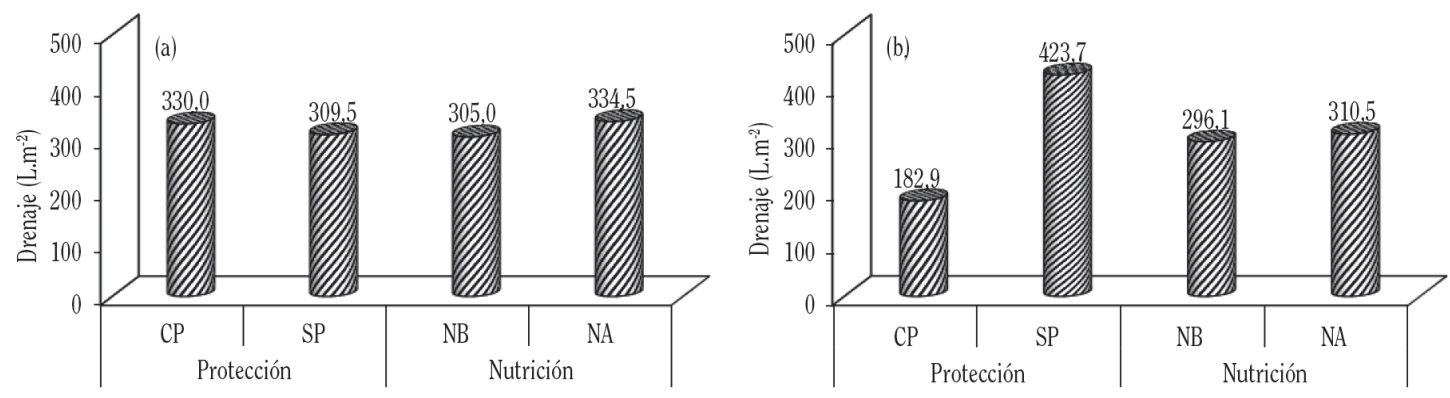

Figura 9. Volúmenes de drenaje total durante (a) la etapa I, después de trasplante, en época seca y (b) etapa II, después de la primera cosecha, en época lluviosa, de un cultivo de culantro coyote hidropónico, bajo condición protegida (CP) y sin protección (SP), con baja (NB) y alta (NA) concentración de nutrientes.

Alajuela, Costa Rica. 2016.

En los diferentes periodos decenarios de ambas etapas de cultivo, la EUA no fue afectada por la interacción $(\mathrm{p}>0,05)$ entre protección $\mathrm{y}$ nutrición, sin embargo, ambos factores afectaron $(p<0,05)$ individualmente, con diferente magnitud, según la etapa de cultivo. En la etapa I (Figura 8a), las mayores EUA se obtuvieron a los 50 DDT, con incrementos de $60 \%$, debido a la protección, y de
$67 \%$ al aumentar el nivel de nutrición. En la etapa II (Figura 8b), las máximas EUA se obtuvieron a los 40 DDT, periodo en el cual se presentaron incrementos de $59 \%$ y $41 \%$, debido a la protección $\mathrm{y}$ al incremento de la nutrición, respectivamente.

Drenaje. En ambas etapas de cultivo, el volumen de drenaje no fue afectado por la 
interacción $(p>0,05)$ entre ambas condiciones de protección y de nutrición. En la etapa I únicamente hubo efecto individual $(\mathrm{p}<0,05)$ del nivel de nutrición (Figura 9a), mientras que en etapa II solamente afectó $(p<0,05)$ la condición de protección (Figura 9b). En la etapa I de cultivo (Figura 9a), el volumen de drenaje incrementó un $9 \%$ al aumentar la concentración de nutrientes (NA). En contraste, en la etapa II (Figura 9b) el volumen de drenaje aumentó apreciablemente un $132 \%$ al pasar de una condición CP a otra SP.

\section{DISCUSIÓN}

En el cultivo en culantro coyote convencional, en suelo a campo abierto, se reportan bajos rendimientos; lo cual hace ineficiente el uso de los recursos, entre ellos el agua y los fertilizantes. El presente estudio demostró que el cultivo hidropónico protegido representa una excelente alternativa de producción, ya que incrementa sustancialmente los rendimientos y reduce el consumo hídrico, de esta manera mejora así la EUA. Sin embargo, la principal limitación para implementar el cultivo hidropónico de culantro coyote, es la poca información sobre parámetros de uso de agua para el manejo del riego, tales como la $\mathrm{ET}_{\mathrm{c}} \mathrm{y} \mathrm{K}_{\mathrm{c}}$, que permita realizar un UEA, basado en altos rendimientos y en la reducción de la $\mathrm{ET}_{\mathrm{c}}$. El rendimiento y la $\mathrm{ET}_{\mathrm{c}}$ a la vez dependen del clima, genética y de las prácticas de manejo del cultivo. En este estudio, se manipularon 2 prácticas de manejo, la protección y la nutrición, que afectaron los diferentes parámetros de uso de agua y el rendimiento, que están directamente relacionadas con la EUA.

Evapotranspiración, rendimiento y eficiencia de uso de agua. En ambas etapas de cultivo, la mayor EUA se obtuvo bajo condiciones protegidas $(\mathrm{CP})$ debido a una reducción de la $\mathrm{ET}_{\mathrm{c}}$ y con mayor aporte de nutrientes (NA) producto de un incremento en el rendimiento. En la etapa I de cultivo en época seca, a pesar de que los rendimientos fueron similares en ambas condiciones ambientales, al reducir la radiación solar (Rs) en el cultivo protegido $(\mathrm{CP})$ con sarán, la $\mathrm{ET}_{\mathrm{c}}$ disminuyó, y como consecuencia aumentó la EUA. En la presente investigación, la DLI se redujo un promedio de $58 \%$ al pasar de un cultivo SP a una condición protegida.

Al respecto, muchos estudios han demostrado una alta relación entre Rs y $\mathrm{ET}_{\mathrm{c}}$ en diferentes cultivos (Fan et al. 2016, Martín et al. 2005), ya que a mayores valores de radiación, mayores requerimientos de agua, como por ejemplo frijol a campo abierto (Escalante et al. 2001) y chile dulce hidropónico en invernadero (Fernández et al. 2005). Específicamente en culantro coyote, Callejas et al. (2016) y Sosa (2006) demostraron que un cultivo protegido con $40 \%$ de sombra obtuvo rendimientos superiores al cultivo sin sombra, lo cual probablemente al estar relacionado con una menor $\mathrm{ET}_{\mathrm{c}}$ se traduce en mayor EUA.

Al analizar el efecto de la nutrición sobre la EUA, independientemente de la protección, en ambas etapas de cultivo, esta incrementó al subir el nivel de nutrición. Esta mayor EUA con NA, contrario al efecto de protección, se debió al incremento del rendimiento, ya que la $\mathrm{ET}_{\mathrm{c}}$ no fue afectada al aumentar el aporte de nutrientes. Relacionado con lo anteriror, Fallovo et al. (2009) y Hoque et al. (2010) demostraron que conforme aumentó el aporte de nutrientes en el cultivo de lechuga, incrementó el rendimiento fresco.

El rendimiento fresco en la segunda etapa del cultivo en época lluviosa, fue superior al obtenido en la etapa I de cultivo en época seca. Esto probablemente fue debido a que, en etapa I, la planta recién trasplantada tiene un periodo de establecimiento de su sistema radical y de formación de fuente (índice de área foliar: IAF) para acumular suficientes reservas para los ciclos de producción posteriores. En todos los cultivos multicosechas o perennes, es normal que exista una curva de rendimiento, donde la producción inicial es baja e incrementa progresivamente hasta alcanzar un pico de máxima producción en cosechas posteriores y una disminución en periodo de senescencia. Lo anterior, se evidenció en el comportamiento del IAF (datos no reportados), el cual mostró un apreciable incremento 
a partir de los 30 DDT en etapa I, y después de 15 DDT en etapa II, hasta alcanzar valores máximos de 7,0 (50 DDT) y de 14,0 (40 DDC), respectivamente.

Drenaje y evapotranspiración. El volumen de agua drenada es una consecuencia de las prácticas de manejo del riego y la nutrición del cultivo, y de las condiciones ambientales (CP y $\mathrm{SP})$. En ambas etapas, el drenaje estuvo relacionado con los demás componentes del balance hídrico, que incluye el aporte total de agua (riego + precipitación) y la $\mathrm{ET}_{\mathrm{c}}$. Las variaciones en los aportes de agua (riego y precipitación) y en la $\mathrm{ET}_{\mathrm{c}}$, repercutieron directamente sobre el comportamiento del volumen drenado, tal como se discute a continuación.

En la etapa I de cultivo, en época seca, el drenaje únicamente fue afectado por el nivel de nutrición. La protección no afectó, ya que el aporte total de agua fue similar en ambas condiciones de protección $(\mathrm{CP}, \mathrm{SP})$, puesto que el sarán es un material permeable que redujo levemente $(15 \%)$ la entrada de lluvia. Consecuentemente, bajo saran (CP), comparado al cultivo SP, hubo un ligero incremento del drenaje (5\%) relacionado con una reducción en la $\mathrm{ET}_{\mathrm{c}}$. Respecto al efecto de la nutrición, aun cuando el aporte de agua fue similar en ambas condiciones de nutrición, el incremento en la nutrición (NA) redujo la $\mathrm{ET}_{\mathrm{c}}$ en un $10 \%$, lo cual se reflejó en un incremento similar en el volumen drenado (10\%).

En la etapa II del cultivo en época lluviosa, por el contrario, el volumen drenado solamente fue afectado por la protección con techo plástico, debido a que el cultivo SP recibió mayor aporte de agua (103\%) como resultado de la alta precipitación. Consecuentemente, el drenaje en el cultivo SP aumentó en mayor magnitud (132\%) que la $\mathrm{ET}_{\mathrm{c}}(44 \%)$, comparado con el cultivo bajo plastico (CP). Otro aspecto que pudo contribuir al incremento de la $\mathrm{ET}_{\mathrm{c}}$ en el cultivo $\mathrm{SP}$ fue un menor efecto osmótico sobre el consumo de agua, ya que presentó una menor $\mathrm{CE}$ en el medio de cultivo, debido al lavado de sales por la alta precipitación.
En el cultivo de culantro coyote, no se reportan estudios acerca del efecto de la sanillidad sobre parámetros de uso de agua. No obstante, diversos estudios en otros cultivos de hoja han demostrado que altos niveles de CE generan un desbalance del potencial osmótico en el medio de cultivo, y consecuentemente, inhiben la absorción de agua (Pessarakli 1999, Taiz y Zieger 2002, Calori et al. 2014). En una revisión de la tolerancia a la salinidad en diferentes cultivos hortícolas, Shannon y Grieve (1998) demostraron que el rendimiento disminuyó entre un 8 y 16\%, cuando la CE superó umbrales de 2,5 dS.m ${ }^{-1}$. Por otra parte, Putra y Yuliando (2015) indicaron que en producción hidropónica, valores elevados de $\mathrm{CE}$ afectan el consumo de agua del cultivo.

Por otra parte, en la etapa II del cultivo en época lluviosa, independientemente de la protección, la ausencia de efecto del nivel de nutrición sobre la $\mathrm{ET}_{\mathrm{c}}$ probablemente estuvo relacionada con el efecto osmótico resultante de una alta CE al incrementar la concentración de sales con NA y su consecuente efecto sobre el consumo de agua del cultivo. Los valores de CE en el agua de drenaje alcanzaron incrementos de hasta $154 \%$ y $282 \%$ en las etapas I y II, respectivamente, al aumentar el aporte de nutrientes con NA.

$\mathrm{El} \mathrm{K}_{\mathrm{c}}\left(\mathrm{ET}_{\mathrm{c}} / \mathrm{ET}_{\mathrm{o}}\right)$ considera las características morfo-fisiológicas del cultivo, las cuales varían según la técnica de producción, el genotipo, el clima y las prácticas de manejo agronómico. Al respecto, algunos estudios han demostrado que los $\mathrm{K}_{\mathrm{c}}$ en chile dulce bajo invernadero se reducen apreciablemente respecto a los $\mathrm{K}_{\mathrm{c}}$ de FAO-56 (Soto 2019, Fernández et al. 2005), relacionado con una reducción de la radiación solar y a cambios en la arquitectura y distribución del dosel vegetal.

El $\mathrm{K}_{\mathrm{c}}$ es un parámetro indispensable para estimar los requerimientos hídricos de los cultivos $\left(\mathrm{ET}_{\mathrm{c}}\right)$ como el producto de la $\mathrm{ET}_{\mathrm{o}} \mathrm{x} \mathrm{K}_{\mathrm{c}}$ (Allen et al. 2006), lo cual contribuye a optimizar los aportes de agua y consecuentemente a mejorar la UEA. La limitante de dicho método radica en que en muchas regiones del mundo no existen datos locales de $\mathrm{K}_{\mathrm{c}}$, ante lo cual normalmente se 
utilizan valores del manual FAO-56 (Allen et al. 2006). Sin embargo, los $\mathrm{K}_{\mathrm{c}}$ FAO-56 fueron obtenidos en diferentes condiciones de clima, manejo y genotipo, por lo que su utilización en el trópico podría conllevar a imprecisiones en el aporte de agua a los cultivos.

Además, actualmente no existen datos de $\mathrm{K}_{\mathrm{c}}$ para el cultivo de culantro coyote en suelo $\mathrm{y}$ menos para el cultivo hidropónico. Esto evidencia la necesidad de generar $\mathrm{K}_{\mathrm{c}}$ para condiciones de cultivo que representen sus características morfo-fisiológicas, de acuerdo con el genotipo, el clima y el manejo agronómico. En el presente estudio, no hubo interacción ni efectos de la protección y la nutrición sobre los $\mathrm{K}_{\mathrm{c}}$. De acuerdo con las curvas típicas de $\mathrm{K}_{\mathrm{c}}$ FAO-56 descritas por Allen et al. (2006), para otros cultivos de hoja, se esperaría un $\mathrm{K}_{\mathrm{c}}$ inicial bajo $(0,2$ a 0,3$)$, producto de una menor $\mathrm{ET}_{\mathrm{c}}$ respecto a la $\mathrm{ET}_{\mathrm{o}}$, debido a la poca transpiración inicial relacionado con un bajo IAF. Posteriormente, el $\mathrm{K}_{\mathrm{c}}$ incrementa linealmente durante la etapa de desarrollo hasta alcanzar valores máximos, coincidente con la etapa de maximo crecimiento del cultivo. Sin embargo, los valores de $\mathrm{K}_{\mathrm{c}}$ FAO-56 no consideran el efecto las prácticas de manejo (protección, riego, nutrición, podas, deshojas, etc.), la técnica de cultivo (campo abierto, invernadero, en suelo o sin suelo), sobre la distribución de la evaporación y la transpiración como componentes de la $\mathrm{ET}_{\mathrm{c}}$.

El manejo del riego en cultivos en sustrato es diferente al de suelo, debido a las diferencias en cuanto a volumen disponible y las características físicas e hidráulicas. En el sistema hidropónico utilizado en este estudio, el manejo del riego influyó en una mayor $\mathrm{ET}_{\mathrm{c}}$ al inicio del cultivo, debido a un alto componente de evaporación inicial. En este sistema, se utilizó un programa de riegos por tiempos, con gran número de riegos cortos y frecuentes, que favoreció una alta evaporación desde la superficie húmeda y expuesta del sustrato en etapas iniciales del cultivo. De esta forma, la $\mathrm{ET}_{\mathrm{c}}$ inicial fue ligermanete inferior a la $\mathrm{ET}_{\mathrm{o}}$, por lo que, consecuentemente, el $\mathrm{K}_{\mathrm{c}}$ fue de 0,9 . Posteriormente, conforme el cultivo crece
$(>\mathrm{IAF})$ y aumenta la cobertura verde sobre la superficie del sustrato, la evaporación disminuye e incrementa la transpiración que pasa a ser el componente mayoritario de la $\mathrm{ET}_{\mathrm{c}}$.

\section{CONCLUSIONES}

El sistema de cultivo hidropónico representa una excelente alternativa al sistema de producción convencional en suelo y campo abierto, ya que en ambas etapas del cultivo presentaron una alta EUA, debido a una apreciable reducción de la $\mathrm{ET}_{\mathrm{c}}$ bajo protección con sarán en época seca y con plástico en época lluviosa y a un sustancial incremento en los rendimientos bajo condiciones protegidas y con un mayor nivel de nutrición. Los rendimientos en dichas condiciones de manejo, en etapas I y II, respectivamente, fueron equivalentes a 18 y 40 rollos $\mathrm{m}^{2}$ con mayor nivel de nutrición, y a 16 y 35 rollos $\mathrm{m}^{2}$ bajo protección. Al considerar que la EUA es resultado del cociente entre rendimiento y $\mathrm{ET}_{\mathrm{c}}$, la mayor EUA bajo protección $(\mathrm{CP})$ fue debido a una reducción sustancial de la $\mathrm{ET}_{\mathrm{c}}$, ya que el rendimiento fue similar en ambas condiciones ambientales.

En ambas etapas de cultivo, la protección redujo apreciablemente la $\mathrm{ET}_{\mathrm{c}}$ del cultivo, ya que disminuyó la radiación solar incidente y, consecuentemente, la evaporación al incio del cultivo cuando había baja cobertura y la transpiración en el resto del ciclo del cultivo. La superior EUA, en el cultivo con mayor nivel de nutrición, fue debido a un incremento en el rendimiento, ya que la $\mathrm{ET}_{\mathrm{c}}$ fue similar en ambos niveles de nutrición, probablemente, debido al efecto osmótico de una mayor concentración de sales.

Las variaciones en el volumen drenado se dieron en función del aporte total de agua y de la $\mathrm{ET}_{\mathrm{c}}$, lo cual dependió de la condición ambiental (CP, SP) y del nivel de nutrición. En la etapa I del cultivo protegido con sarán, el drenaje se redujo cuando el nivel de nutrición fue menor, debido a que la $\mathrm{ET}_{\mathrm{c}}$ fue mayor y los aportes de agua similares. En la etapa II de cultivo protegido con techo plástico, el drenaje disminuyó 
apreciablemente, ya que evitó aportes excesivos de agua provenientes de la lluvia y redujo la $\mathrm{ET}_{\mathrm{c}}$. La disponibilidad de $\mathrm{K}_{\mathrm{c}}$ específicos para el cultivo de culantro coyote hidropónico, según genotipo, manejo del cultivo y clima, representan parámetros útiles para mejorar la UEA. Este estudio, por primera vez, reporta valores de $\mathrm{K}_{\mathrm{c}}$ para el cultivo de culantro coyote bajo un manejo hidropónico en condiciones protegidas y un nivel de nutrición alto, que demostró altos rendimientos y una mejora en la EUA comparado al cultivo sin protección.

\section{LITERATURA CITADA}

Allen, RG; Pereira, LS; Raes, D; Smith, M. 2006. Evapotranspiración del cultivo. Guías para la determinación de los requerimientos de agua de los cultivos. Estudio FAO-56. Riego y Drenaje. Roma, Italia. $323 \mathrm{p}$.

Alvarado, Y; Sanabria, C; Villalobos, J. 1999. El cultivo de culantro coyote (Eringyum foetidum) para exportación. San José, Costa Rica, Ministerio de Agricultura y Ganadería. 17 p.

Ansorena, MJ. 1994. Sustratos. Propiedades y caracterización. Madrid, España, Mundi Prensa. 167 p.

Bertsch, F. 2003. Absorción de nutrimentos por los cultivos. San José, Costa Rica, Asociación Costarricense de la Ciencia del Suelo. 308 p.

Callejas, I; Cerritos, R; Rauda, M. 2016. Caracterización morfoagronómica e invertebrados asociados al acapate (Apaiaceae: Eryngium foetidum L.). Facultad de Ciencias Agronómicas. Universidad de El Salvador, El Salvador. 97 p.

Calori, A; Factor, T; Lima Júnior, S; Moraes, I; Barbosa, P; Tivelli, S; Purquerio, L. 2014. Condutividade elétrica da solução nutritiva e espaçamento entre plantas na produção de beterraba e alface. Hortic. Brasileira 32(4):426-433.

Cerdas, M; Montero, M. 2016. Efecto de cambios en manejo poscosecha sobre la calidad y vida útil del culantro coyote para exportación. Agronomía Costarricense 40(1):51-64.

Christiansen, JE. 1942. Irrigation by Sprinkling. California Agricultural Experiment Station. USA, Bulletin $\mathrm{N}^{\mathrm{o}}$ 670. $126 \mathrm{p}$.

Di Rienzo, JA; Macciavelli, RE; Casanoves. F. 2012. Modelos Lineales Mixtos: aplicaciones en InfoStat. Universidad de Córdoba, Córdoba, Argentina. 193 p.

Elliott, J; Deryng, D; Müller, C; Frieler, K; Konzmann, M; Gerten, D; Glotter, M; Flörke, M; Wada, Y; Best, N; Eisner, S; Fekete, BM; Folberth, C; Foster, I; Gosling, SN; Haddeland, I; Khabarov, N; Ludwig,
F; Masaki, Y; Olin, S; Rosenzweig, C; Ruane, AC; Satoh, Y; Schmid, E; Stacke, T; Tang, Q; Wisser, D. 2014. Constraints and potentials of future irrigation water availability on agricultural production under climate change. Proceedings of the National Academy of Sciences 111(9):3239-3244.

Escalante, J; Escalante, I; Rodríguez, M. 2001. Producción de fríjol, en dos épocas de siembra: su relación con la evapotranspiracion, unidades calor y radiación solar en clima cálido. Terra Latinoamericana 19(4):309-315.

Eunice, E. 2006. Evaluación de la producción de culantro coyote (Eryngium foetidum) en tres ambientes diferentes y dos tipos de fertilización en la zona Atlántica de Costa Rica. Tesis Lic. Limón, Costa Rica, Universidad EARTH. 41 p.

Fallovo, C; Rouphael, Y; Rea, E; Battistelli, A; Colla, G. 2009. Nutrient solution concentration and growing season affect yield and quality of Lactuca sativa $\mathrm{L}$. var. acephala in floating raft culture. J. Sci. Food Agric. 89(10):1682-1689.

Fan, J; Wu, I; Zhang, T; Xiang, Y; Zheng, J. 2016. Climate change effects on reference crop evapotranspiration across different climatic zones of China during 1956-2015. J. Hydrol. 542:923-937.

Fernández, MD; Gallardo, M; Bonachela, S; Orgaz, F; Thompson, RB; Fereres, E. 2005. Water use and production of a greenhouse pepper crop under optimum and limited water supply. J. Hortic. Sci. Biotech. 80(1):87-96.

Hoque, M; Ajwa, H; Othman, M; Smith, R; Cahn, M. 2010. Yield and postharvest quality of lettuce in response to nitrogen, phosphorus, and potassium fertilizers. Hort. Sci. 45(10):1539-1544.

Jara, D. 2016. Evaluación de tres híbridos de pimiento (Capsicum annum L.) cultivados en hidroponía con tres mezclas de sustrato. Tesis Ing. Agr. Universidad de Guayaquil. Guayaquil, Ecuador. 46 p.

Lavagni, G; Rodríguez, G. 2009. El Culantro Coyote, producto de exportación. Alimentaria 102:30-36.

Leite, C. 2005. Las mallas en el contexto del proyecto de un vivero. Hortic. Internacional 1:66-73.

Martín, F; López, P; Calera, A. 2005. Agua y Agronomía. Madrid, España, Mundi-Prensa. 496 p.

Morales, J; Brunner, B; Flores, 1; Martínez, S. 2013. Hoja Informativa: culantro orgánico. Proyecto de agricultura orgánica. Departamento de cultivos y ciencias agroambientales. Estación Experimental Agrícola de Lajas, Lajas, Puerto Rico. 11 p.

Oren-shamir, M; Gussakovsky, E; Shpiegel, E; Nissim-levi, A; Ratner, K; Ovadia, R; Giller, Y; Shahak, Y. 2001. Coloured shade nets can improve the yield and quality of green decorative branches of Pittosporum variegatum. J. Hortic. Sci. Biotech. 76(3):353-361. 
Pessarakli, M. 1999. Handbook of plant and crop stress. 2 ed. Nueva York, Estados Unidos, Marcel Dekker, Inc. $1188 \mathrm{p}$.

Putra, P; Yuliando, H. 2015. Soilless culture system to support water use efficiency and product quality: a review. Agriculture and Agricultural Science Procedia 3:283-288.

Resh, H. 2001. Cultivos hidropónicos, nuevas técnicas de producción. 5a edición. Madrid, España, MundiPrensa. 547 p.

Rodríguez, DA; Hoyos, M; Chang, M. 2001. Soluciones nutritivas en hidroponía. Formulación y preparación. $2^{\text {a }}$ edición. Lima, PE, Universidad Agraria La Malina, Centro de Investigación de Hidroponía e Investigación Mineral. 97 p.

Rojas, J; Paniagua, F. 2015. Comportamiento agronómico de Capsicum annuum L., Lycopersicon esculentum M. y Cucumis melo L. bajo cultivo protegido hidropónico utilizando la solución universal de Steiner. Tesis Lic. San Carlos, Costa Rica, Tecnológico de Costa Rica. 98 p.

Santos, L. 2001. La producción de recao o culantro (Eryngium foetidum L.) en Puerto Rico (en línea). Rio Piedras, Puerto Rico. Consultado 20 jun. 2017. Disponible en https://issuu.com/estacion/docs/technologicalpackage---coriander

SFE (Servicio Fitosanitario del Estado). 2014. Informe de resíduos de plaguicidas en vegetales del año 2014. San José, Costa Rica, Departamento de agroquímicos y equipos. $9 \mathrm{p}$.

Shahak, Y; Gal, E; Offir, Y; Ben-Yakir, D. 2008. Photoselective shade netting integrated with greenhouse technologies for improved performance of vegetable and ornamental crops. Acta Hortic. 797:75-80.

Shahak, Y; Gussakovsky, E; Cohen, Y; Lurie, S; Stern, R; Kfir, S; Naor, A; Atzmon, J; Doron, I; GreenblatAvron, Y. 2004. Colornets: a new approach for light manipulation in fruit trees. Acta Hortic. 636:609-616.
Shannon, M; Grieve, C. 1998. Tolerance of vegetable crops to salinity. Sci.Hortic. 78(1-4):5-38.

Silva, V de P DA; Tavares, Al; Sousa, IF. 2013 Evapotranspiração e coeficientes de cultivo simples e dual do coentro. Hortic. Brasileira 31(2):255-259.

Sosa, E. 2006. Evaluación de la producción de culantro coyote (Eryngium foetidum Lineo) en tres ambientes diferentes y dos tipos de fertilización en la zona atlántica de Costa Rica. Tesis Lic. Guácimo, Costa Rica, Universidad EARTH. 55 p.

Soto, F. 2018. Parámetros para el manejo del agua en tomate y chile dulce hidropónico bajo invernadero. Agronomía Costarricense 42(2):59-73.

Soto, F. 2019. Coeficientes de cultivo según densidad de siembra y volumen de sustrato en chile dulce bajo invernadero. Pesquisa Aplicada \& Agrotecnologia 12(1):07-21.

Soto, F; Ramírez, A. 2005. Hidroponía. Centro Nacional Especializado Granja Modelo. San José, Costa Rica, Instituto Nacional de Aprendizaje. 109 p.

Taiz, L; Zeiger, E. 2002. Plant Physiology. 3 ed. Sunderland, Massachusetts, USA, Sinauer Associates, Inc 764 p.

Tilman, D; Cassman, KG; Matson, PA; Naylor, R; Polasky, S. 2002. Agricultural sustainability and intensive production practices. Nature 418(6898):671-677.

UNE (Una Norma Española)-EN (European Norm)-13041. 2007. Mejoradores de suelo y sustratos de cultivo. Determinación de las propiedades físicas: densidad aparente seca, volumen de aire, volumen de agua, valor de contracción y porosidad total. Madrid, España, Asociación Española de Normalización y Certificación (AENOR). 24 p.

Urrestarazu, GM. 2015. Manual práctico del cultivo sin suelo e hidroponía. Almería, España, MundiPrensa. $278 \mathrm{p}$.

WWAP (United Nations World Water Assessment Programme). 2016. The United Nations World Water Development Report 2016: Water and Jobs. Paris, Francia, UNESCO. 148 p.

Todos los derechos reservados. Universidad de Costa Rica. Este artículo se encuentra licenciado con Creative Commons Reconocimiento-NoComercial-SinObraDerivada 3.0 Costa Rica. Para mayor información escribir a rac.cia@ucr.ac.cr 